\title{
Reduction of Complex Energy-Integrated Process Networks using Graph Theory
}

\author{
Sujit S. Jogwar* \\ Department of Chemical Engineering \\ Institute of Chemical Technology, Mumbai, 400019, India \\ Srinivas Rangarajan, Prodromos Daoutidis \\ Department of Chemical Engineering and Materials Science, \\ University of Minnesota, Minneapolis, MN 55455, USA
}

\begin{abstract}
This paper focuses on the analysis of complex (multi-loop) energyintegrated process networks. Simple (single-loop) energy-integrated networks (comprising of large energy recycle or throughput) with twotime scale dynamics are the building blocks for such complex networks. The modular structure of these complex networks lends them to a graph theoretic analysis, whereby weak and strong connections between process units arising from time scale separation are identified from structural information. Subsequently, a graph-theoretic framework for network analysis and control is developed, and connecting links are built to an equivalent analysis using singular perturbations. The proposed analysis framework is illustrated via application to a representative complex process network.
\end{abstract}

\section{Introduction}

Aggressive efforts in sustainability and improved energy efficiency in the process industries have led to the development of complex process designs with multiple energy integration loops. While these integrated systems have demonstrated significant economic benefits, they have had limited practical implementation owing to the difficulties in their operation and control. Specifically, these integrated networks exhibit complex, nonlinear, multitime scale dynamics with strong interactions among process units (Jacobsen, 1999; Kumar and Daoutidis, 2002; Jogwar et al., 2009). These interactions limit the effectiveness of standard decentralized controllers. Fully centralized controllers, though possible to design, are impractical due to the large

\footnotetext{
${ }^{*}$ Corresponding author: Telephone:

(9122) 33612033, email: ss.jogwar@ictmumbai.edu.in
} 
size of the plant model and are often ill-conditioned showing strong sensitivity to modeling errors (Larsson et al., 2003; Kiss et al., 2005; Kumar and Daoutidis, 2002).

Recently, network-level analysis approaches have been developed to address this problem in a systematic and generic manner. These approaches include:

- Passivity-based control (Ydstie, 2002; Hudson and Bao, 2012; Hioe et al., 2013; Dörfler et al., 2009) which involves determining the passivity/dissipativity of a process unit using thermodynamic properties, and analyzing the stability properties of the network based on the interconnections of these passive/dissipative components.

- Plantwide control which involves taking a holistic approach in terms of identifying control objectives, manipulated inputs and selecting a control structure for a network. Though some of the earlier contributions in this area were heuristics-based (Luyben et al., 1997; Stephanopoulos and Ng, 2000), recent works (Zhu et al., 2000; Skogestad, 2004) have added formalism to this approach.

- Distributed control (Venkat et al., 2008; Rawlings and Stewart, 2008; Liu et al., 2009) which places itself between the two extremes - decentralized and fully centralized control schemes. This approach involves designing controllers for subsystems (group of units) and allowing for information sharing (and possibly control/optimization objectives) among these controllers.

- Quasi-decentralized control (El-Farra et al., 2005; Sun and El-Farra, 2008; Baldea et al., 2013) which incorporates a local control system for each unit in a network along with a supervisory unit at the network level, and these local systems communicate with the supervisor as well as the other local systems through a shared communication medium.

- Hierarchical control (Scattolini, 2009; Kumar and Daoutidis, 2002; Jogwar et al., 2009) which aims at decomposing the control problem into different tiers, typically based on the corresponding time scales. The control objectives at a unit level are addressed by the lower tier whereas the objectives at the network level are addressed by the top tier.

In previous work (Jogwar et al., 2010), we have identified two classes of energy-integrated networks, one characterized by the presence of large energy recycle (Figure 1(a)) and the other characterized by the presence of large energy throughput (Figure 1(b)), which are at the core of most energy-integrated networks. These networks show two-time scale dynamics and naturally lend themselves to a two-tiered control approach based on 
these time scales. Furthermore, we have shown that the underlying energy flow structure of a complex energy-integrated network consists of interconnections of these recycles and throughputs (Jogwar, 2011). For example, a network of distillation columns separating a five-component mixture (Figure 2) has been shown to involve six recycles and three throughputs with energy flows of 3 different orders of magnitude (see Jogwar and Daoutidis (2010) for details). A reactor-heat exchanger network designed for naphtha reforming (Figure 3) has also been shown to be composed of 9 recycles and 10 throughputs, with the various energy flows spanning three orders of magnitude (see Jogwar (2011) for details).

It can be reasonably argued that the multi-loop integrated structure of a complex process network has the potential to demonstrate multi-time scale dynamics, and the dynamic characteristics exhibited by the parent complex network can be inferred from the properties of its building blocks. Furthermore, the interconnection of (a large number of) these building blocks can result in interactions and dynamic phenomena being exhibited by these networks that are more intricate than can be anticipated by considering the mere association of these building blocks. The development of a model reduction and control framework for such complex networks, building upon the results obtained for simple networks is the main objective of this paper.

In section 2, we develop a singular perturbations-based reduction framework for a generic complex energy-integrated process network. Singular perturbations have been used extensively for model reduction and control applications (O'Malley Jr., 1991; Ladde and Siljak, 1983; Desoer and Shahruz, 1986; Khalil and Kokotovic, 1979; Kokotovic, 1981; Kokotovic et al., 1986; Dmitriev and Kurina, 2006). The key distinguishing feature of the systems considered is that they are (typically) in a non-standard singularly perturbed form. Such systems have received much less attention in the literature, especially in the case of multiple (more than two) time scales (Fenichel, 1979; Marino and Kokotovic, 1988; Kumar et al., 1998; Vora et al., 2006). The model reduction framework is subsequently exploited to develop a graphbased reduction framework which is the main contribution of this paper. In section 3, we describe this graph-theoretic formulation to capture and automate the major reduction steps in the analysis of complex energy-integrated process networks. The advantages of the graph-based reduction framework over the model-based singular perturbations approach are also highlighted. The proposed methods are illustrated with the help of a complex process network. Preliminary results on this work were presented in Jogwar et al. (2011); Heo et al. (2012). Applications of the presented algorithm to complex energy-integrated systems have been described in Heo et al. (2014). 


\section{Analysis of Complex Energy-Integrated Net- works}

Let us consider a generic complex energy-integrated network, consisting of $N$ units (indexed by $j$ ) and energy (enthalpy) flows, $h_{i}$, spanning $m$ orders of magnitude (indexed by $i$ ). The energy flows of different order of magnitude are segregated from each other through the definition of small parameters $\varepsilon_{i}$, such that $\varepsilon_{i} \ll 1$ and $\frac{\varepsilon_{i+1}}{\varepsilon_{i}} \ll 1$ (with $\varepsilon_{0} \approx 1$ ). Nominal steady state flows of each magnitude are used to define these small parameters such that $\varepsilon_{i}=\frac{h_{0, s}}{h_{i, s}}$, where subscript $s$ denotes a steady state value. In energyintegrated systems, the small parameters $\varepsilon_{i}$ represent the ratios of liquid to vapor enthalpy (in the case of a combined boiler-condenser configuration), small and large material flows (in the case of large reflux ratio) and/or the combination of these (in the case of heat pump operation for high purity separation).

For each unit, we consider the enthalpy change due to flow in/out from other units and external energy flows $d_{j}$ (representing contributions from heat of reaction, compressor work, etc). These energy flows are scaled using the nominal steady state flows and the corresponding energy balance equations can be represented in the following form:

$$
\frac{d \mathbf{H}}{d t}=\sum_{i=0}^{m-1} \frac{1}{\varepsilon_{i}} \mathbf{F}_{i} \mathbf{g}_{i}\left(\mathbf{H}, \mathbf{u}_{i}\right)
$$

$\mathbf{H}$ is the vector of enthalpies of each of the $N$ units in the network. $\mathbf{g}_{i}$ are vectors of dimension $p_{i}$ corresponding to the contributions from the energy flows of order $\mathcal{O}\left(\frac{1}{\varepsilon_{i}}\right)$. $\mathbf{F}_{i}$ (of dimension $N \times p_{i}$ ) represents the corresponding selector matrix such that $F_{i}(j, k)$ is 1 if the energy balance equation of the enthalpy $H_{j}$ contains the term $g_{i}(k)$ and all the other elements of $\mathbf{F}$ are $0 . \mathbf{u}_{i}$ are scaled energy flow variables which can be used as potential manipulated inputs. Note that Eq. (1) results from an energy balance under the assumption that the contributions of kinetic and potential energy are very small compared to internal energy and can therefore be neglected, and the fact that at constant temperature and pressure, the rate of change of enthalpy and internal energy are the same.

Eq. (1) is a singularly perturbed system with multiple singular perturbation parameters in a non-standard form, potentially leading to energy dynamics evolving over $m$ time scales (Vora et al., 2006). Due to the lack of explicit separation of the state variables according to the time scale they evolve in Eq. (1), a unit enthalpy may evolve in multiple time scales. To uncover this hierarchy of time scales of unit enthalpies, we successively use singular perturbations to derive approximate reduced order models of the dynamics in the different time scales, starting with the fastest (corresponding to the largest energy flows). 
Defining a stretched time scale $\tau_{m-1}=\frac{t}{\varepsilon_{m-1}}$, the dynamic equations (1) become:

$$
\frac{d \mathbf{H}}{d \tau_{m-1}}=\sum_{i=0}^{m-1} \frac{\varepsilon_{m-1}}{\varepsilon_{i}} \mathbf{F}_{i} \mathbf{g}_{i}\left(\mathbf{H}, \mathbf{u}_{i}\right)
$$

In the limit $\varepsilon_{m-1} \rightarrow 0$, the description of the dynamics in the fastest time scale is given by:

$$
\frac{d \mathbf{H}}{d \tau_{m-1}}=\mathbf{F}_{m-1} \mathbf{g}_{m-1}\left(\mathbf{H}, \mathbf{u}_{m-1}\right)
$$

as $\forall i \neq(m-1), \frac{\varepsilon_{m-1}}{\varepsilon_{i}} \rightarrow 0$ as $\varepsilon_{m-1} \rightarrow 0$.

Note that all the enthalpies in a network may not be affected by the largest magnitude energy flows. This means $\mathbf{F}_{m-1}$ does not necessarily have a full row rank. Premultiplying Eq. (2) by $\mathbf{F}_{m-1}^{T}$, we obtain the simplified description of the fast dynamics as:

$$
\frac{d \mathbf{H}_{m-1}}{d \tau_{m-1}}=\left(\mathbf{F}_{m-1}^{T} \mathbf{F}_{m-1}\right) \hat{\mathbf{g}}_{m-1}\left(\mathbf{H}_{m-1}, \mathbf{u}_{m-1}\right)
$$

where $\mathbf{H}_{m-1}=\mathbf{F}_{m-1}^{T} \mathbf{H}$ represent the enthalpies evolving in this fast time scale and $\hat{\mathbf{g}}_{m-1}$ represents the $\mathbf{g}_{m-1}$ vector in terms of $\mathbf{H}_{m-1}$.

$$
\mathbf{g}_{m-1}\left(\mathbf{H}, \mathbf{u}_{m-1}\right)=\hat{\mathbf{g}}_{m-1}\left(\mathbf{H}_{m-1}, \mathbf{u}_{m-1}\right)
$$

Note that only the energy flows $\mathbf{g}_{m-1}$ of order $\mathcal{O}\left(\frac{1}{\varepsilon_{m-1}}\right)$ govern the dynamics in this time scale. This fast dynamics converges to a quasi-steady state given by the following constraints:

$$
0=\left(\mathbf{F}_{m-1}^{T} \mathbf{F}_{m-1}\right) \hat{\mathbf{g}}_{m-1}\left(\mathbf{H}_{m-1}, \mathbf{u}_{m-1}\right)
$$

or simply

$$
0=\hat{\mathbf{g}}_{m-1}\left(\mathbf{H}_{m-1}, \mathbf{u}_{m-1}\right)
$$

as the matrix $\left(\mathbf{F}_{m-1}^{T} \mathbf{F}_{m-1}\right)$ is nonsingular. Differentiating the constraints (6) with respect to t, we obtain:

$$
0=\frac{\partial \hat{\mathbf{g}}_{m-1}}{\partial t}=\frac{\partial \hat{\mathbf{g}}_{m-1}}{\partial \mathbf{H}_{m-1}} \frac{d \mathbf{H}_{m-1}}{d t}
$$

At this stage, there are two cases depending on the nature of the constraints in Eq. (6).

- Case 1 - Linearly independent constraints:

In this case, the matrix $\frac{\partial \hat{\mathbf{g}}_{m-1}}{\partial \mathbf{H}_{m-1}}$ is non-singular and thus Eq. (7) results in $\frac{d \mathbf{H}_{m-1}}{d t}=0$. This means that the enthalpies $\mathbf{H}_{m-1}$ evolve only in 
this fast time scale $\tau_{m-1}$. The energy dynamic equations after this fast dynamics are given by:

$$
\frac{d \mathbf{H}}{d t}=\sum_{i=0}^{m-2} \frac{1}{\varepsilon_{i}} \hat{\mathbf{F}}_{i} \mathbf{g}_{i}\left(\mathbf{H}, \mathbf{u}_{i}\right)
$$

where $\hat{\mathbf{F}}_{i}$ represent the correspondingly adjusted selector matrices, taking into the account the fact that the enthalpies $\mathbf{H}_{m-1}$ do not evolve in the slower time scales.

- Case 2 - Linearly dependent constraints:

In this case, the matrix $\frac{\partial \hat{\mathbf{g}}_{m-1}}{\partial \mathbf{H}_{m-1}}$ is singular. This means that the fast dynamics for the enthalpies $\mathbf{H}_{m-1}$ are accompanied by evolution in the slower time scales too. Let us define a matrix $\mathbf{B}_{m-1}$ of dimension $\left(p_{m-1} \times q_{m-1}\right)$ such that

$$
0=\mathbf{g}_{m-1}\left(\mathbf{H}, \mathbf{u}_{m-1}\right)=\mathbf{B}_{m-1} \tilde{\mathbf{g}}_{m-1}\left(\mathbf{H}, \mathbf{u}_{m-1}\right)
$$

wherein $\tilde{\mathbf{g}}_{m-1}\left(\mathbf{H}, \mathbf{u}_{m-1}\right)=0$ represent the linearly independent set of constraints. The description of the energy dynamics after this fast boundary layer is obtained by taking the limit $\varepsilon_{m-1} \rightarrow 0$ in the original time scale $t$, and is given as:

$$
\begin{aligned}
\frac{d \mathbf{H}}{d t} & =\sum_{i=0}^{m-2} \frac{1}{\varepsilon_{i}} \mathbf{F}_{i} \mathbf{g}_{i}\left(\mathbf{H}, \mathbf{u}_{i}\right)+\mathbf{F}_{m-1} \mathbf{B}_{m-1} \lim _{\varepsilon_{m-1} \rightarrow 0} \frac{\tilde{\mathbf{g}}_{m-1}\left(\mathbf{H}, \mathbf{u}_{m-1}\right)}{\varepsilon_{m-1}} \\
0 & =\tilde{\mathbf{g}}_{m-1}\left(\mathbf{H}, \mathbf{u}_{m-1}\right)
\end{aligned}
$$

In this limiting case, the term $\lim _{\varepsilon_{m-1} \rightarrow 0} \frac{\tilde{\mathbf{g}}_{m-1}\left(\mathbf{H}, \mathbf{u}_{m-1}\right)}{\varepsilon_{m-1}}$ becomes indeterminate. However, this term is finite and represents the difference between large $\mathcal{O}\left(\frac{1}{\varepsilon_{m-1}}\right)$ terms. Let's denote it by $\mathbf{z}_{m-1}$. The resulting energy dynamics is now given as:

$$
\begin{aligned}
\frac{d \mathbf{H}}{d t} & =\sum_{i=0}^{m-2} \frac{1}{\varepsilon_{i}} \mathbf{F}_{i} \mathbf{g}_{i}\left(\mathbf{H}, \mathbf{u}_{i}\right)+\mathbf{F}_{m-1} \mathbf{B}_{m-1} \mathbf{z}_{m-1} \\
0 & =\tilde{\mathbf{g}}_{m-1}\left(\mathbf{H}, \mathbf{u}_{m-1}\right)
\end{aligned}
$$

This is a differential algebraic equation (DAE) system. It can be verified (see Kumar et al. (1998) for the required conditions) that this is an index-2 DAE and the "algebraic" variables $\mathbf{z}_{m-1}$ can be obtained after differentiating the constraints in Eq. (10) with respect to time, as

$$
\begin{gathered}
\mathbf{z}_{m-1}=-\left(\mathcal{L}_{\mathbf{F}_{m-1} \mathbf{B}_{m-1}} \tilde{\mathbf{g}}_{m-1}\left(\mathbf{H}, \mathbf{u}_{m-1}\right)\right)^{-1} \times \\
{\left[\sum_{i=0}^{m-2} \frac{1}{\varepsilon_{i}} \mathcal{L}_{\mathbf{F}_{i} \mathbf{g}_{i}} \tilde{\mathbf{g}}_{m-1}\left(\mathbf{H}, \mathbf{u}_{m-1}\right)\right]}
\end{gathered}
$$


The Lie derivative notation $\mathcal{L}$ is used to represent derivatives along the flow of a vector. For a vector $\mathbf{h}(\mathbf{x})$ and a matrix (or a vector) $\mathbf{f}(\mathbf{x})$, Lie derivative $\mathcal{L}_{\mathbf{f}(\mathbf{x})} \mathbf{h}(\mathbf{x})$ is defined as:

$$
\mathcal{L}_{\mathbf{f}(\mathbf{x})} \mathbf{h}(\mathbf{x})=\mathbf{J}_{\mathbf{h}} \mathbf{f}
$$

with $\mathbf{J}_{\mathbf{h}}=\left\{\frac{\partial h_{i}}{\partial x_{j}}\right\}$ representing the jacobian matrix of $\mathbf{h}$. Eq. (11) can also be represented as a linear combination of $\mathcal{O}(1)$ terms multiplied by $1 / \varepsilon_{i}$.

$$
\mathbf{z}_{m-1}=\sum_{i=0}^{m-2} \frac{1}{\varepsilon_{i}} \overline{\mathbf{z}}_{i}
$$

where $\overline{\mathbf{z}}_{i}$ represents the terms in $\mathbf{z}_{m-1}$ corresponding to the energy flows of order $\mathcal{O}\left(\frac{1}{\varepsilon_{i}}\right)$. Substituting for $\mathbf{z}_{m-1}$ in Eq. (10), the energy dynamics after the fast boundary layer is given by:

$$
\begin{aligned}
\frac{d \mathbf{H}}{d t} & =\sum_{i=0}^{m-2} \frac{1}{\varepsilon_{i}}\left[\mathbf{F}_{i} \mathbf{g}_{i}\left(\mathbf{H}, \mathbf{u}_{i}\right)+\mathbf{F}_{m-1} \mathbf{B}_{m-1} \overline{\mathbf{z}}_{i}\right] \\
0 & =\tilde{\mathbf{g}}_{m-1}\left(\mathbf{H}, \mathbf{u}_{m-1}\right)
\end{aligned}
$$

Eq. (13) can be represented in the following condensed form:

$$
\begin{aligned}
\frac{d \mathbf{H}}{d t} & =\sum_{i=0}^{m-2} \frac{1}{\varepsilon_{i}} \hat{\mathbf{F}}_{i} \hat{\mathbf{g}}_{i}\left(\mathbf{H}, \mathbf{u}_{i}\right) \\
0 & =\tilde{\mathbf{g}}_{m-1}\left(\mathbf{H}, \mathbf{u}_{m-1}\right)
\end{aligned}
$$

Note that the dynamic equations after the fast boundary layer [Eq. (8) \& (14)] are similar to the original dynamic equations Eq. (1) and thus, this model reduction procedure can be performed repeatedly for each of the slower time scales, defined by $\tau_{i}=\frac{t}{\varepsilon_{i}}$, to obtain the entire hierarchy of models in the different time scales.

Remark 2.1 It has been previously shown that large energy throughputs result in linearly independent constraints (Jogwar et al., 2010). Therefore, if the largest energy flows in a network form a throughput structure, the constraints (6) will be independent and the results for Case 1 will apply. Similarly, if the largest energy flows form a recycle structure, the constraints (6) will be dependent (Jogwar et al., 2009) and the results for Case 2 will apply.

Remark 2.2 For the case of a single large recycle loop corresponding to the time scale $\tau_{m-1}$ (similar arguments will hold for the subsequent time scales), 
we can note that the total enthalpy of the recycle loop $\left(H_{\text {total }, m-1}\right)$ does not depend on the large internal flows. Specifically,

$$
\frac{d H_{\text {total }, m-1}}{d \tau_{m-1}}=\sum_{k=1}^{p_{m-1}} \frac{d \mathbf{H}_{m-1}[k]}{d \tau_{m-1}}=0
$$

resulting in

$$
\sum_{k=1}^{p_{m-1}} \mathbf{g}_{m-1}[k]=0
$$

where $\mathbf{H}_{m-1}[k]$ and $\mathbf{g}_{m-1}[k]$ represent the $k$ th elements of the vectors $\mathbf{H}_{m-1}$ and $\mathbf{g}_{m-1}$, respectively. This means that only $\left(p_{m-1}-1\right)$ constraints (out of $\left.p_{m-1}\right)$ in $\mathbf{g}_{m-1}$ are independent, giving rise to a one-dimensional equilibrium manifold and a one-dimensional slower time scale dynamics which is captured by the total enthalpy of the recycle loop. This also implies that the individual components of the large recycle evolve in the fast time scale whereas the total enthalpy of the loop evolves only in the slower time scales.

We can arbitrarily set the last constraint equation in (6) as the linear combination of the rest of the $\left(p_{m-1}-1\right)$ constraints, thus resulting in

$$
\mathbf{B}_{m-1}=\left(\begin{array}{c}
\mathbf{I}_{\left(p_{m-1}-1\right) \times\left(p_{m-1}-1\right)} \\
-\mathbf{1}_{1 \times\left(p_{m-1}-1\right)}
\end{array}\right)
$$

with $\mathbf{I}$ being the identity matrix and $\mathbf{1}$ being a matrix with all entries equal to 1. Once the structure of the matrix $\mathbf{B}_{m-1}$ is set, Eq. (10) represents the description of slower time scale dynamics in a DAE form.

Note that a complex network can involve multiple independent (disconnected) large recycle loops (for example, see Figure 5) and the dimension of the corresponding slow dynamics will be equal to the multiplicity of such independent loops.

Remark 2.3 For a recycle, if the inputs and outputs are of the same order of magnitude as that of the recycle (as shown in Figure 4), the corresponding constraints in (6) are linearly independent and the treatment is identical to the case of throughput. Furthermore, it can be easily shown that the constraints (6) are linearly independent for the cases of splitting and mixing (including bypass). The mathematical treatment, for these cases, is therefore identical to the case of a throughput.

The analysis presented above shows that a complex network with multiple energy integration loops can be sequentially analyzed by identifying the energy flow structures with similar magnitude flows. Additionally, the properties of the prototype configurations (large recycle, large throughput) can be applied to these structures to identify dynamic characteristics in that 
time scale. Motivated by this, we next develop a graph-theoretic framework to systematically perform such a network reduction in an automated and scalable manner.

\section{Graph-theoretic reduction framework}

Let us first introduce a graph-theoretic formalism to represent energy flows in process networks by modifying some graph theory terminologies. Specifically, we define:

- Energy flow graph: A weighted graph $\mathcal{G}(N, E)$ of the energy flows in a process network - the nodes $\mathrm{N}$ represent individual process entities (e.g. heat exchanger passes, trays of a distillation column) and the edges $\mathrm{E}$ (directed and weighted) represent the direction and the order of magnitude of the energy flows. Sources and sinks are not explicitly represented. Directed edges with no tail (or head) node are energy flows from sources (or to sinks).

- Energy flow path: An open walk of nodes and edges of the energy flow graph, starting from an energy source and terminating at an energy sink, such that no node is repeated.

- Energy flow cycles: A closed walk of nodes and edges such that no node, except the first node, is repeated.

- Induced subgraph: For an energy flow graph $\mathcal{G}(N, E)$, for a given order of magnitude ' $\mathrm{m}$ ' of the energy flows, $\mathcal{H}_{m}$ represents an induced subgraph such that it contains all \& only the energy flows of magnitude 'm'.

Let us now consider the energy flow graphs corresponding to the two building blocks - recycles and throughputs. In the energy flow graphs, throughputs are energy flow paths, while recycles are energy flow cycles. These blocks can be replaced in the graph by a single composite unit to reduce the complexity of the representation and, as will be seen later in the paper, also to analyze the dynamics of the energy balance variables in the process network. Figure 1(b) shows a large throughput of energy over $\mathrm{N}$ units starting from a source supplying energy at a rate $Q_{i n}$ and ending with a sink taking energy at a rate $Q_{\text {out }}$. Note that energy flows of different orders of magnitude are distinguished by lines of different thickness. The equivalent representation is a unit labeled $T_{L(N)}^{\mathcal{O}\left(h_{1}\right)}$. $T$ denotes throughput, the superscript represents the order of magnitude of the energy flows within the block and the subscript is a set of labels of the units constituting the composite unit (for example, $L(N)=\{1,2, \ldots, N\}$ ). Figure 1(a) shows a simple network with large recycle and its equivalent representation as a unit 
labeled $R_{L(N)}^{\mathcal{O}\left(h_{1}\right)}$. Again, $R$ represents recycle, the superscript represents the order of magnitude of energy recycle and the subscript represents the set of labels of the units in the recycle.

Based on the results for these prototype networks, it can be established that:

- all the units of the throughput block in Figure 1(b) evolve in a fast time scale corresponding to $\mathcal{O}\left(h_{1}\right)$ energy flows. This time scale can be expressed by stretching the original time $t$ by a small term $\varepsilon_{1}=\frac{h_{0, s}}{h_{1, s}}$ as $\tau_{1}=\left(\frac{h_{1, s}}{h_{0, s}}\right) t$,

- all the constituents of the recycle block in Figure 1(a) evolve in the fast time scale $\tau_{1}$, while the the overall enthalpy of the recycle block evolves in the slow time scale $t$.

Let us now consider the energy flow graph representation of the complex network analyzed in section 2. For every order of magnitude $i$ exhibited by the energy flows of the network, we can find an induced subgraph denoted as $\mathcal{H}_{i}$. This subgraph can consist of several connected components, each one of which can be a throughput (energy flow path), recycle (energy flow cycle), or a combination of these. Based on remark 2.3, if cycles are contained within a path, the whole component can be considered as a path. For the highest magnitude energy flows, we can obtain an induced subgraph $\mathcal{H}_{m-1}$ with ' $\psi$ ' connected components. In this case, we can write:

$$
\mathbf{g}_{m-1}\left(\mathbf{H}, \mathbf{u}_{m-1}\right)=\left(\begin{array}{c}
\mathbf{g}_{m-1}^{1}\left(\mathbf{H}, \mathbf{u}_{m-1}^{1}\right) \\
\vdots \\
\mathbf{g}_{m-1}^{\psi}\left(\mathbf{H}, \mathbf{u}_{m-1}^{\psi}\right)
\end{array}\right)
$$

Note that we can always rearrange the elements of $\mathcal{H}_{m-1}$ to allow for such grouping of $\mathbf{g}_{m-1}\left(\mathbf{H}, \mathbf{u}_{m-1}\right)$. For the fastest time scale $\tau_{m-1}$, we have:

$$
\frac{d \mathbf{H}}{d \tau_{m-1}}=\mathbf{F}_{m-1}\left(\begin{array}{c}
\mathbf{g}_{m-1}^{1}\left(\mathbf{H}, \mathbf{u}_{m-1}^{1}\right) \\
\vdots \\
\mathbf{g}_{m-1}^{\psi}\left(\mathbf{H}, \mathbf{u}_{m-1}^{\psi}\right)
\end{array}\right)
$$

which can be decomposed into ' $\psi$ ' groups as:

$$
\frac{d \mathbf{H}^{\kappa}}{d \tau_{m-1}}=\mathbf{F}_{m-1} \mathbf{g}_{m-1}^{\kappa}\left(\mathbf{H}, \mathbf{u}_{m-1}^{\kappa}\right), \forall \kappa=1,2, \ldots, \psi
$$

Each component $\kappa$ is independent and we can establish the time scale properties of these enthalpies based on the nature (cycle/path) of these individual connected components. Figure 5 shows an example with two connected 
components in the energy flow graph corresponding to the largest magnitude energy flows. Such an energy flow structure represents two tightly energy-integrated processes connected weakly via a material throughput stream. In this case, the two recycles can be analyzed independently in the time scale corresponding to the largest magnitude energy flows. Here, $\mathbf{H}^{1}=\left\{H_{1}, H_{2}, H_{3}, H_{4}\right\}$ and $\mathbf{H}^{2}=\left\{H_{5}, H_{6}, H_{7}, H_{8}\right\}$.

Remark 3.1 A pure recycle (no incoming/outgoing edges) can also occur as a combination of several connected (fused) cycles that share at least one node (e.g. Figure 6(a), obtained in a vapor-recompression distillation network (Jogwar and Daoutidis, 2010)). For a component $i$ with pure recycle, we have

$$
\mathbf{g}_{m-1}^{\kappa}\left(\mathbf{H}, \mathbf{u}_{m-1}^{\kappa}\right)=\mathbf{S u}_{m-1}^{\kappa}
$$

where $\mathbf{S}$ is the incidence matrix of the component $\kappa$ of $\mathcal{H}_{m-1}$. The rank of the incidence matrix with $r$ rows and components is $r-c$ (Chung, 1997). Thus we have:

$$
\operatorname{rank}(\mathbf{S})=\mathcal{N}_{m-1}^{\kappa}-1
$$

where $\mathcal{N}_{m-1}^{\kappa}$ is the number of nodes in the component $\kappa$. Thus, irrespective of the number of cycles, the dynamics of the connected cycles is similar to that of pure recycle with a one-dimensional equilibrium manifold. It can be easily verified that this slow variable represents the total enthalpy of this component.

Remarks 2.3 and 3.1 allow us to propose a graph reduction strategy wherein combinations of recycles and throughputs can be condensed to an equivalent cycle or a path. Two such example graphs are shown in Figure 6. These energy flow graphs represent components of real integrated systems, in this case, side rectifiers and heat pump assisted distillation columns (Jogwar and Daoutidis, 2010). The energy flow graphs of such networks can be simplified by identifying the throughputs and the recycles in the system and replacing them with their equivalent representations (see Figure 1). Figure 6(a) shows the simplification of a graph with multiple cycles to a single (recycle) unit. Figure 6(b) shows that two paths branching out from a single node of the process graph (splitting case referred to in remark 2.3) will also ultimately simplify to a single (throughput) unit. Note that a composite recycle (or throughput) block will exhibit the same time scale multiplicity properties as a simple recycle (or throughput) block.

Based on these arguments, we propose the following algorithm to identify dynamic time scales, classify control objectives and obtain reduced order representations of dynamic equations in each time scale for a complex network. 
ComplexNetworkAnalysis $(\mathcal{G}, W)$

1: Sort $\mathrm{W}$ in descending order

2: for $i=1$ to $\operatorname{Size}(\mathrm{W})=m$ do

3: $\quad \mu=\mathrm{W}[\mathrm{i}]$

4: $\quad \mathcal{H}_{\mu}=\operatorname{InducedSubgraph}(\mathcal{G}, \mu)$

5: $\quad\left[F_{\mu}, \mathbf{g}_{\mu}\left(\mathbf{H}_{\mu}, \mathbf{u}_{\mu}\right)\right]=\operatorname{Ebalance}\left(\mathcal{H}_{\mu}\right)$

6: $\quad \mathcal{T}\left(\tau_{\mu}\right)=$ nodes $\in \mathcal{H}_{\mu}$

7: $\quad$ for each node $\mathrm{N} \in \mathcal{H}_{\mu}$ do

8: $\quad$ if $\mathrm{N}$ is a composite node then

9: $\quad$ add $\sum N[k]$ to $\mathcal{Y}\left(\tau_{\mu}\right)$

10: $\quad$ else

11:

12 :

13:

add $\mathrm{N}$ to $\mathcal{Y}\left(\tau_{\mu}\right)$

$$
\text { end if }
$$

end for

$\mathcal{U}\left(\tau_{\mu}\right)=$ Edges in $\mathcal{H}_{\mu}$

$\mathcal{C}=$ SmallestElementaryCycle $\left(\mathcal{H}_{\mu}\right)$

while $\mathcal{C} \neq \phi$ do

$\operatorname{GraphReduce}(\mathcal{G}, \mathcal{C}, \mu)$

GraphReduce $\left(\mathcal{H}_{\mu}, \mathcal{C}, \mu\right)$

$\mathcal{C}=$ SmallestElementaryCycle $\left(\mathcal{H}_{\mu}\right)$

end while

if size(RecycleTimes) $\neq 0$ then

$D A E_{\mu}=\mathbf{F}_{\mu^{-}} \mathbf{B}_{\mu^{-}} \overline{\mathbf{z}}_{\mu}$

Constraint $\mu_{\mu}=\tilde{\mathbf{g}}_{\mu^{-}}\left(\mathbf{H}_{\mu^{-}}, \mathbf{u}_{\mu^{-}}\right)$

if size(RecycleTimes) $>1$ then

AddConstraints ${ }_{\mu}=\sum_{j=1}^{\text {size(RecycleTimes })} D A E_{j}$

\section{end if}

end if

if degree $(\mathrm{N})=0$ for any node $\mathrm{N} \in \mathcal{H}_{\mu}$ then

Add $\tau_{\mu}$ to RecycleTimes

Add $N_{j}$ to PureRecycles

All but 1 out of $N_{j}$ should be controlled in this time scale

end if

if degree $(\mathrm{N}) \neq 0$ for all nodes $\mathrm{N} \in \mathcal{H}_{\mu}$ then

Clear RecycleTimes, PureRecycles

end if

for all node $\mathrm{N} \in \mathcal{H}_{\mu}$ such that degree $(\mathrm{N}) \neq 0$ do

if $\mathrm{N}$ is a composite node then

Remove $N[k]$ from PureRecycles end if 
40: $\quad$ Remove $\mathrm{N}$ from $\mathcal{G}$

41: end for

42: $\quad$ Reduced order model in $\tau_{\mu}, \mathcal{D}_{\mu}$, is

43:

$$
\begin{aligned}
\frac{d \mathbf{H}_{\mu}}{d \tau_{\mu}} & =h_{1, s} \times\left[\mathbf{F}_{\mu} \mathbf{g}_{\mu}\left(\mathbf{H}_{\mu}, \mathbf{u}_{\mu}\right)+D A E_{\mu}\right] \\
0 & =\text { Constraint }_{\mu}+\text { AddConstraints } \text { Ans }_{\mu}
\end{aligned}
$$

44: end for

45: Overall energy balance equations $(\mathcal{D})$ are

46:

$$
\frac{d \mathbf{H}}{d t}=h_{1, s} \times \sum_{\mu=W[1]}^{W[m]} \frac{1}{\varepsilon_{\mu}} \mathbf{F}_{\mu} \mathbf{g}_{\mu}\left(\mathbf{H}, \mathbf{u}_{\mu}\right)
$$

47: $\operatorname{return} \mathcal{T}, \mathcal{Y}, \mathcal{U}, \mathcal{D}, \mathcal{D}_{\mu}$

The inputs to the algorithm are the graph $\mathcal{G}(\mathrm{N}, \mathrm{E})$ and a vector $\mathrm{W}$ of the various orders of magnitude exhibited by different energy flows. The outputs of the algorithm are

- $\mathcal{T}$, a set such that $\mathcal{T}\left(\tau_{\mu}\right)$ refers to the set of enthalpies (nodes) evolving in the time scale $\tau_{\mu}$.

- $\mathcal{Y}$, a set such that $\mathcal{Y}\left(\tau_{\mu}\right)$ refers to the set of variables (nodes) to be controlled in the time scale $\tau_{\mu}$.

- $\mathcal{U}$, a set such that $\mathcal{U}\left(\tau_{\mu}\right)$ refers to the set of manipulated inputs (edges) to be used in the time scale $\tau_{\mu}$.

- $\mathcal{D}$, a stiff, multi-timescale dynamic model for energy dynamics

- $\mathcal{D}_{\mu}$, a reduced order model for energy dynamics in timescale $\tau_{\mu}$

Figure 7 shows the flowchart for the algorithm depicting the major steps. Since one seeks the evolution of the system for times $t=0 \rightarrow \infty$, the algorithm begins with the largest order of magnitude energy flows (corresponding to the fastest time scale) and proceeds to the smallest.

For a given order of magnitude $\mu$, a subroutine InducedSubgraph generates a subgraph $\mathcal{H}_{\mu}$ from the parent graph $\mathcal{G}$ such that all the edges in $\mathcal{H}_{\mu}$ are of the order $\mu$. The subroutine Ebalance generates the components of scaled energy balance equations corresponding to the contributions of the energy flows of order $\mu$. This subroutine employs traversing algorithms (Cormen, 2001) (for example, depth-first search, breadth-first search, etc.) to extract the information regarding connectivity between different nodes which can be used to reconstruct the corresponding vectors for the scaled 
energy balance equations. For example, in Figure 5, using a graph traversing algorithm, one can establish, graphically, that node 4 is connected to nodes 2,3 , and 5 , with the edge $2-4$ (of order $\mathcal{O}(1 / \varepsilon)$ ) entering the node and the edges 4-3 $(\mathcal{O}(1 / \varepsilon))$ and $4-5(\mathcal{O}(1))$ leaving the node. Thus the energy balance equation for the enthalpy of node $4\left(H_{4}\right)$ will involve contributions from $\mathbf{g}_{1}$ and $\mathbf{g}_{0}$. Furthermore, the selector matrix $\mathbf{F}_{\mu}\left(\right.$ size $\left.N \times N_{\mu}\right)$ can be constructed such that $F_{j k}=1$ if node $j$ represents the $k$ th node in the subgraph $\mathcal{H}_{\mu}$. As the dynamics of the nodes in $\mathcal{H}_{\mu}$ are affected by energy flows of the order $\mu$, they evolve at the time scale $\tau_{\mu}=\frac{t}{\varepsilon_{\mu}}$. All these nodes are added to the set $\mathcal{T}\left(\tau_{\mu}\right)$. Consequently, all the edges in $\mathcal{H}_{\mu}$ are considered potential manipulated inputs in this time scale and thus, are added to the set $\mathcal{U}\left(\tau_{\mu}\right)$.

In order to proceed with the graphical model reduction (as depicted in Figure 6(a)), recycle structures need to be identified in the subgraph $\mathcal{H}_{\mu}$. These recycles represent 'large energy recycle' as viewed from a slower time scale. As recycles represent cycles in the subgraph, the first step is to identify cycles in $\mathcal{H}_{\mu}$. The subroutine SmallestElementaryCycle $\left(\mathcal{H}_{\mu}\right)$ finds the smallest cycle in $\mathcal{H}_{\mu}$. We use elementary circuit detection because we don't want repetition of nodes. Elementary cycles in a graph can be obtained by efficient algorithms (Ehrenfeucht et al., 1973; Tarjan, 1973; Johnson, 1975). As there can be multiple cycles in a subgraph, the reduction algorithm needs to systematically lump the integrated cycles and separately keep the isolated cycles. For example, the structure in Figure 6(a) results in two elementary cycles, however, these are integrated and represent a single large energy recycle loop. On the other hand, the structure in Figure 5 also contains two elementary cycles representing two large recycle loops. The reduction operation is performed using the while loop (as shown in Figure 6(a)). Once a cycle is found, the subroutine $\operatorname{GraphReduce}\left(\mathcal{H}_{\mu}, \mathcal{C}, \mu\right)$ replaces the nodes of $\mathcal{C}$ in $\mathcal{H}_{\mu}$ with a composite node labeled $R_{L}^{\mu}$ where $\mathrm{L}$ is the set of nodes in $\mathcal{C}$. Graph rewriting algorithms (Dörr, 1995; Heckel, 2006) are available to implement such a replacement procedure. The iteration (the while loop) continues until all the cycles in $\mathcal{H}_{\mu}$ are replaced by composite nodes. Here, $\phi$ represents an empty set. At this stage, $\mathcal{H}_{\mu}$ contains only composite recycle nodes and/or throughputs. Note that the same reduction is performed for the parent graph $\mathcal{G}$. In a particular time scale, all the constituent units of a given recycle/throughput block of a process network have the same dynamic properties. Therefore, upon simplification, it is sufficient to retain the information of the nodes and the order of magnitude of the energy flow cycles and paths of $\mathcal{H}_{\mu}$ (or $\mathcal{G}$ ). This is the reason of using the subroutine GraphReduce on the parent graph.

Since the energy dynamics of all the units of a throughput block evolve in a single time scale, these units need not be considered while analyzing the subsequent slower time scales. Therefore the algorithm seeks all the 
nodes with edges $(\operatorname{degree}(\mathrm{N}) \neq 0)$, as they would necessarily be a part of a throughput, and removes them all from $\mathcal{G}$ (line 40). Removal of a node leads to edges without a tail (or head) node. These edges represent energy flows which have negligible (compared to the large throughput) contribution to the energy dynamics of the throughput. They can be treated as energy flows from the source (or to the sink) without any loss of information regarding the energy dynamics.

The dependence/independence of quasi-steady state constraints arising from a fast (and any subsequent intermediate) time scale plays a key role in the model reduction procedure based on singular perturbations (see section 2 for details). The graph reduction procedure allows us to predict if these constraints are linearly dependent/independent. For example, if the reduced form of a fast subgraph does not have any inputs or outputs, the corresponding quasi-steady state constraints will be linearly dependent. This naturally follows from the fact that the dynamics of this composite node is not affected by any of the order $\mu$ flows (and is probably affected by some of the lower order flows). Such dependent constraints result in DAE descriptions of the slower time scale dynamics (like Eq. (10)). To this end, the if loop between lines 28 to 32 identifies such 'pure recycles' (with zero degree) in a time scale and adds that time scale to the set RecycleTimes. It also adds the component nodes of that recycle $\left(N_{i}\right)$ to the set PureRecycles. While analyzing the slower time scale corresponding to the energy flows of the same order of magnitude as the inlet/outlet of this recycle node, there will be a DAE contribution of the form $\mathbf{F}_{\mu^{-}} \mathbf{B}_{\mu^{-}} \overline{\mathbf{z}}_{\mu}$. Such a time scales (represented as $\mu^{-}$) is identified using the if loop between lines 21 to 27 by searching for such 'pure recycles' in previous time scales. Here, $\overline{\mathbf{z}}_{\mu}$ represent the terms in $\mathbf{z}_{\mu^{-}}$corresponding to the energy flows of order of magnitude $\mu$ (see Eq. (12) for details). The algebraic variables $\mathbf{z}_{\mu^{-}}$are given by $\lim _{\varepsilon_{\mu^{-}} \rightarrow 0} \frac{\tilde{\mathbf{g}}_{\mu^{-}}\left(\mathbf{H}_{\mu^{-}}, \mathbf{u}_{\mu^{-}}\right)}{\varepsilon_{\mu^{-}}}$ with $\tilde{\mathbf{g}}_{\mu^{-}}\left(\mathbf{H}_{\mu^{-}}, \mathbf{u}_{\mu^{-}}\right)$being the linearly independent constraints from the previous time scale. The matrix $\mathbf{B}_{\mu^{-}}$relates the original dependent constraints to the independent constraints as:

$$
\mathbf{g}_{\mu^{-}}\left(\mathbf{H}_{\mu^{-}}, \mathbf{u}_{\mu^{-}}\right)=\mathbf{B}_{\mu^{-}} \tilde{\mathbf{g}}_{\mu^{-}}\left(\mathbf{H}_{\mu^{-}}, \mathbf{u}_{\mu^{-}}\right)
$$

Furthermore, the number of linearly independent constraints can be calculated as the difference between the number of nodes in the original subgraph and the number of nodes in the reduced subgraph. This also suggests the evolution of these nodes in the slower time scales. As described in remark 2.2 , selecting the first $\left(p_{\mu^{-}}-1\right)$ independent constraints for each connected 
components, the structural form of the matrix $\mathbf{B}_{\mu^{-}}$is given by:

$$
\mathbf{B}_{\mu^{-}}=\left[\begin{array}{lll}
\ddots & & \\
& \mathbf{I}_{\left(p_{\mu^{-}}^{\kappa}-1\right) \times\left(p_{\mu^{-}}^{\kappa}-1\right)} & \\
& (-1) \mathbf{1}_{1 \times\left(p_{\mu^{-}}^{\kappa}-1\right)} & \\
& & \ddots
\end{array}\right]
$$

The matrix $\mathbf{F}_{\mu^{-}}$is the selector matrix as identified earlier (for a faster time scale) using the subroutine Ebalance. A (large) recycle may be a part of another recycle in a slower time scale. The if loop between lines 24 to 26 accounts for contributions due to such recycles spanning multiple time scales. This will continue till such recycles are a part of a throughput, in which case, there is no further slower time scale dynamic contribution due to these recycles. The if loop between lines 33 to 35 ensures that the effect of recycle is limited only till a throughput is encountered.

The multi-time scale dynamics allows for the development of a hierarchical control strategy with the control objectives in each time scale being addressed at separate tiers. The subgraph $\mathcal{H}_{\mu}$ (before graph reduction) is needed for identifying control objectives and manipulated inputs in a time scale. Specifically, all the simple (non-composite) nodes in this subgraph represent nodes evolving in that time scale and thus their enthalpy should be controlled in that time scale. A composite recycle node in this subgraph represents a slower time scale contribution from a recycle in a faster time scale. Thus the total enthalpy of this recycle block should be controlled in this time scale (see Remark 2.2). The if loop between lines 8 to 12 essentially performs this operation. Additionally, a comment on line 31 states that in the case of a network with large recycle ('pure recycles'), the enthalpies of all the component nodes cannot be simultaneously controlled in the fast time scale as the total enthalpy evolves in the slower time scale.

The canonical form of the reduced order model (similar to Eq. (2), $\mathcal{D}_{\mu}$, capturing the dynamics of energy balance variables in a particular time scale $\left(\tau_{\mu}\right)$ is given by the addition of the contributions from the energy flows of order $\mu\left(\mathbf{g}_{\mu}\left(\mathbf{H}_{\mu}, \mathbf{u}_{\mu}\right)\right)$ and any DAE contributions from recycles in a faster time scale. Note that, for the case of a 'pure recycle' in a faster time scale, $\mathbf{F}_{i}$ and $\mathbf{g}_{i}$ in Eq. (2) are given by $\hat{\mathbf{F}}_{i}$ and $\hat{\mathbf{g}}_{i}$ as defined by Eq. (13) and (14).

The procedure described above is repeated for the subsequent orders of magnitude present in $\mathrm{W}$. The canonical form of the overall (multi-timescale) dynamic equations (similar to Eq. (1)), $\mathcal{D}$, is constructed by the 'weighted' sum of contributions from energy flows of each order of magnitude.

Remark 3.2 The recycles are identified prior to throughputs, because identifying and removing throughputs first leads to ambiguity in differentiating between a recycle and a bypass block involving some of the units of a throughput. As can be been in Figure 4, if the throughputs are identified before the 
recycles, both the recycle and the bypass are erroneously represented by the same composite block. On the other hand, if the recycles are identified before the throughputs, the recycle and the bypass have the different reduced forms.

Remark 3.3 This algorithm captures the two-time scale dynamics of the prototype network with large energy recycles by identifying that the units of the recycle block of larger magnitude evolve in the fast time scale while the composite unit (PureRecycle), representing the enthalpy of the entire recycle block, evolves in the slower time scale.

Remark 3.4 This algorithm generates only the scaled energy balance equations. The definitions of the various energy flows (enthalpy of material flows, heat transfer rates, rates of heat generation/consumption due to reactions, etc) via appropriate energy transfer and energy flow correlations in terms of temperature gradients, heat transfer parameters, etc. will be required for the derivation of system-specific dynamic equations in terms of unit enthalpy/temperature.

Remark 3.5 The described algorithm can be divided into three parts - network reduction in each time scale, identifying control and manipulated variables in each time scale, and formulating the scaled dynamic equations. The latter two parts are directly dependent on the number of nodes $N_{\mu}$ and edges $E_{\mu}$ in each induced subgraph. The network reduction, on the other hand, involves: (a) induced subgraph identification, (b) elementary circuit detection, and (c) graph reduction and rewriting. Induced subgraphs can be identified by identifying among all edges those with the appropriate weight, and hence involves $\mathcal{O}\left(E_{\mu}\right)$ operations. Graph reduction depends only on the nodes of the cycle and all edges included within and external to the cycle. This would, in the worst case, scale with the total number of edges and nodes.

The circuit detection algorithm - which determines all the recycles in the process graph - dictates the overall complexity of our algorithm. While Ehrenfeucht's (Ehrenfeucht et al., 1973) has a worst case $\mathcal{O}\left(N_{\mu} \times E_{\mu} \times\left(C_{\mu}+\right.\right.$ 1)) with $C_{\mu}$ being the total number of circuits, Johnson's (Johnson, 1975) algorithm offers $\mathcal{O}\left(N_{\mu}+E_{\mu}\right)\left(C_{\mu}+1\right)$ worst-case efficiency. Given that cycles have to be identified in each time scale, the overall compelxity of this step is $\sum_{\mu} O\left(N_{\mu}+E_{\mu}\left(C_{\mu}+1\right)\right)$, where $\mu$ runs over each scale.

Remark 3.6 The graph-theoretic algorithm does not require the actual values of all the energy flows in the network. If such values are available, energy flows can be directly segregated into sets of different order of magnitude. An energy flow is the product of material flow and the specific enthalpy (which is a function of the physical state of the stream). In the absence of exact energy flow values, the order of magnitude for an energy flow is determined by identifying levels of material flows and the corresponding physical states. 
For example, liquid phase enthalpy at a temperature for a particular stream is an order of magnitude lower than the vapor phase enthalpy at the same temperature. If the energy flow of the liquid stream is considered to be of $\mathcal{O}(1)$, then the energy flow of the vapor stream at the same temperature will be of a higher order of magnitude, say $\mathcal{O}(1 / \varepsilon)$, where $\varepsilon$ is a small number. Energy balance considerations over process units can also be used to identify such order of magnitude of energy flows. These issues are demonstrated in the example considered in the next section.

\section{Example Complex Energy-Integrated Network}

Let us now consider an application of this graph reduction algorithm to the complex network shown in Figure 3. This system has two endothermic reactors (reformers) operating at elevated temperature compared to the feed. Nine process-to-process heat exchangers $\left(P P X_{1}\right.$ through $\left.P P X_{9}\right)$ are used to reduce external energy consumption by transferring energy from a hot effluent stream to the cold inlet stream, and thus each one of these result in one energy recycle loop. The energy flow graph for this network consists of 50 nodes, as tabulated in Table 1.

Table 2 tabulates the nominal values of the duties of these PPXs, along with the heating/cooling duties of the utilities. Note that this is the only information required for the application of the graph-reduction algorithm. The order of magnitude of all the other energy flows are inferred using order of magnitude arguments based on unit-level energy balances. For example, for the heat exchanger $P P X_{2}$ in this system, the only information available is that the rate of heat transfer in this exchanger is large (say $\mathcal{O}(1 / \varepsilon)$, with $\varepsilon=0.1)$. The energy balance across this exchanger sets the hot inlet flow and the cold outlet flow to be $\mathcal{O}(1 / \varepsilon)$. This, however, does not fix the orders of magnitude for the hot exit flow and the cold inlet flow (which can be either $\mathcal{O}(1)$ or $\mathcal{O}(1 / \varepsilon)$ ). The energy balance across the exchanger $P P X_{1}$ provides the solution. It indicates that the energy flows for the cold streams in this exchanger should be of the same order of magnitude owing to the $\mathcal{O}(1)$ energy transfer term. As the energy flow corresponding to the naphtha feed is assumed to be $\mathcal{O}(1)$, the energy flows for the cold streams in $P P X_{1}$ and the remaining energy flows in $P P X_{2}$ are $\mathcal{O}(1)$.

The results from graph-reduction are tabulated in Table 3 . The various energy balance variables evolve over two time scales. Specifically, the enthalpies of the cold and hot sides of $P P X_{6}$ (16 and 17), the heaters $H_{2}$ (21) and $H_{3}(23)$ and the two reformers (22 and 24) evolve only in the fast time scale. It can be noted that these variables are a part of a large throughput from the large heater $H_{2}$ duty to the endothermic heat of reaction in reformer-1 (via reformer-2, $P P X_{6}, H_{2}$ and reformer-1). The enthalpies of the cold and hot sides of $P P X_{2}(3$ and 4$)$, the heater $H_{1}(5)$ and the desul- 
phurisation reactor (6) evolve in both the fast and the slow time scale, owing to the presence of a large recycle loop (via $P P X_{2}, H_{1}$, desulphurisation reactor). All the other energy balance variables evolve only in the slow time scale. The hierarchical control problem can be formulated as:

- Fast time scale control: The temperatures/enthalpies of cold and hot sides of $P P X_{2}(3$ and 4$)$ and $P P X_{6}\left(16\right.$ and 17), the heater $H_{1}(5)$, the desulphurisation reactor (6), the heaters $H_{2}(21)$ and $H_{3}(23)$ and the two reformers (22 and 24) can be controlled in this time scale. Note that one out of the temperatures of cold and hot sides of $P P X_{2}$, the heater $H_{1}$ and the desulphurisation reactor (6) should be left uncontrolled in this time scale. Some of the potential manipulated inputs include the heating duty of $H_{3}(37 \rightarrow 23)$, the heat transfer rate across $\mathrm{PPX}_{2}(4 \rightarrow 3)$ which can be varied using a bypass stream across this exchanger.

- Slow time scale control: The variables evolving in this time scale, as tabulated in Table 3, can be controlled in this tier. Additionally, the total enthalpy of the recycle block comprising of $P P X_{2}, H_{1}$ and desulphurisation reactor should also be controlled in this time scale.

Equation (15) represents the scaled form of the multi-time scale energy balance equations $(\mathcal{D})$.

$$
\frac{d \mathbf{H}}{d t}=\epsilon \mathbf{F}_{0} \mathbf{g}_{0}\left(\mathbf{H}, \mathbf{u}_{0}\right)+\mathbf{F}_{1} \mathbf{g}_{1}\left(\mathbf{H}, \mathbf{u}_{1}\right)+\frac{1}{\epsilon} \mathbf{F}_{2} \mathbf{g}_{2}\left(\mathbf{H}, \mathbf{u}_{2}\right)
$$

The dynamic equations $\left(\mathcal{D}_{2}\right)$ in the fast time scale $\tau_{1}=t / \varepsilon$ are given by Eq. (16).

$$
\frac{d \mathbf{H}_{2}}{d \tau_{1}}=\mathbf{F}_{2} \mathbf{g}_{2}\left(\mathbf{H}, \mathbf{u}_{2}\right)
$$

where $\mathbf{H}_{2}=\left\{H_{3}, H_{4}, H_{5}, H_{6}, H_{16}, H_{17}, H_{21}, H_{22}, H_{23}, H_{24}\right\}$. There are two connected components in this subgraph; one reycle and one throughput. As there is a pure recycle loop, a subset of the constraints from the fast time scale are linearly dependent. The dynamics in the slower time scale $\left(\mathcal{D}_{1}\right)$ is given by Eq. (17).

$$
\begin{aligned}
& \frac{d \mathbf{H}_{1}}{d \tau_{0}}=\mathbf{F}_{1} \mathbf{g}_{1}\left(\mathbf{H}, \mathbf{u}_{1}\right)+\mathbf{F}_{2} \mathbf{B}_{2} \overline{\mathbf{z}}_{1} \\
& \tilde{\mathbf{g}}_{2}\left(\mathbf{H}, \mathbf{u}_{2}\right)=0
\end{aligned}
$$

where $\tilde{\mathbf{g}}_{2}\left(\mathbf{H}, \mathbf{u}_{2}\right)=0$ represents linearly independent constraints. $\mathbf{H}_{1}$ represents the corresponding vector of energy balance variables evolving in this time scale. $\overline{\mathbf{z}}_{1}$ represents algebraic variables, thus making this slow dynamics a DAE system. Note that the exact forms of the various vectors and matrices $\left(\mathbf{F}_{i}, \mathbf{g}_{i}, \mathbf{B}_{i}\right)$ are generated by the code but, for the sake of brevity, are not reproduced here. 


\section{Conclusions}

In this paper, an analysis framework for complex (multi-loop) energyintegrated networks has been developed. Initially, a model reduction framework using singular perturbations is developed to elucidate the underlying dynamic time scale multiplicity. Motivated by the modular structure of these complex networks, a graph theoretic analysis framework is developed, whereby weak and strong connections between process units arising from time scale separation are identified from structural information. The underlying energy flow structure of a complex network is represented by a graph (referred to as energy flow graph) and an algorithm is presented to

- identify various time scales exhibited by the complex network,

- generate scaled equations describing the (energy) dynamics in each time scale, and

- classify various outputs to be controlled in each time scale and identify potential manipulated inputs to address these control objectives.

The graph-based reduction procedure offers significant advantages over the singular perturbation-based approach. Specifically,

1. The singular perturbation approach will result in a system-specific procedure whereas the graph-based procedure is generic and finds application to a wide variety of complex networks.

2. The graph-based approach does not need exact values of energy flows to perform the analysis. Even the knowledge of approximate order of magnitude is sufficient to perform model reduction.

3. While an automated singular perturbations-based reduction (using symbolic computations) will be computationally expensive, the graphbased reduction steps are not computationally expensive and thus this approach is scalable for large networks (see Remark 3.5 for the computational load of the presented algorithm).

The proposed framework is illustrated with the help of an example complex network. The presented algorithm has also been successfully applied to relevant process network examples - a hydrodealkylation of toluene system (Heo et al., 2012), an energy-integrated solid oxide fuel cell system (Heo et al., 2014), and an energy-integrated distillation column system (Heo et al., 2014).

\section{Acknowledgments}

Partial financial support for this work by the National Science Foundation, grant CBET-1133167 and the Government of India Department of Science 
and Technology (DST) INSPIRE scheme (IFA-13 ENG-61) is gratefully acknowledged.

\section{References}

Baldea, M., El-Farra, N. H., and Ydstie, E. B. Dynamics and control of chemical process networks: Integrating physics, communication and computation. Comput. Chem. Eng., 51:42-54, 2013.

Chung, F. R. K. Spectral Graph Theory. 92. CBMS Regional Conference Series in Mathematics, 1997.

Cormen, T. H. Introduction to algorithms. Technical report, MIT Electrical Engineering and computer Science Series, 2001.

Desoer, C. A. and Shahruz, S. M. Stability of nonlinear systems with three time scales. Circ. Syst. Sig. Proc., 5:449-464, 1986.

Dmitriev, M. G. and Kurina, G. A. Singular perturbations in control problems. Automat. Rem. Contr., 67(1):1-43, 2006.

Dörfler, F., Johnsen, J. K., and Allgöwer, F. An introduction to interconnection and damping assignment passivity-based control in process engineering. J. Process Contr., 19(9):1413-1426, 2009.

Dörr, H. Efficient graph rewriting and its implementation. Springer, 1995.

Ehrenfeucht, A., Fosdick, L. D., and Osterweil, L. J. An algorithm for finding the elementary circuits of a directed graph. Technical Report CUCS-024-73, University of Colorado at Boulder, Department of Computer Science, 1973.

El-Farra, N. H., Gani, A., and Christofides, P. D. Fault-tolerant control of process systems using communication networks. AIChE J., 51(6):1665$1682,2005$.

Fenichel, N. Geometric singular perturbation theory for ordinary differential equations. J. Diff. Equat., 31:53-98, 1979.

Heckel, R. Graph transformation in a nutshell. Electronic Notes in Theoretical Comp. Sci., 148(1):187-198, 2006.

Heo, S., Jogwar, S. S., Rangarajan, S., and Daoutidis, P. Graph reduction for hierarchical control of energy integrated process networks. In IEEE 51st Annual Conference on Decision and Control, pages 6388-6393, 2012. 
Heo, S., Jogwar, S. S., Rangarajan, S., and Daoutidis, P. Graph reduction of complex energy-integrated networks: Process systems applications. AIChE J., 60(3):995-1012, 2014.

Hioe, D., Bao, J., and Ydstie, E. B. Dissipativity analysis for networks of process systems. Comput. Chem. Eng., 50:207-219, 2013.

Hudson, N. and Bao, J. Dissipativity-based decentralized control of interconnected nonlinear chemical processes. Comput. Chem. Eng., 45:84-101, 2012 .

Jacobsen, E. W. On the dynamics of integrated plants-non-minimum phase behavior. J. Process Contr., 9(5):439-451, 1999.

Jogwar, S. S. Dynamics and control of energy integrated process networks. PhD thesis, University of Minnesota, May 2011.

Jogwar, S. S. and Daoutidis, P. Energy flow patterns and control implications for integrated distillation networks. Ind. Eng. Chem. Res., 49(17): 8048-8061, 2010.

Jogwar, S. S., Baldea, M., and Daoutidis, P. Dynamics and control of process networks with large energy recycle. Ind. Eng. Chem. Res., 48(13): 6087-6097, 2009.

Jogwar, S. S., Baldea, M., and Daoutidis, P. Tight energy integration: Dynamic impact and control advantages. Comput. Chem. Eng., 34(9): 1457-1466, 2010.

Jogwar, S. S., Rangarajan, S., and Daoutidis, P. Multi-time scale dynamics in energy-integrated networks: A graph theoretic analysis. In IFAC World Congress, volume 18 of 1, pages 6085-6090, 2011.

Johnson, D. B. Finding all the elementary circuits of a directed graph. SIAM J. Comput., 4(1):77-84, 1975.

Khalil, H. and Kokotovic, P. V. Control of linear systems with multiparameter singular perturbations. Automatica, 15:197-207, 1979.

Kiss, A. A., Bildea, C. S., Dimian, A. C., and Iedema, P. D. Design of recycle systems with parallel and consecutive reactions by nonlinear analysis. Ind. Eng. Chem. Res., 44(3):576-587, 2005.

Kokotovic, P. V. Subsystems, time scales and multimodeling. Automatica, 17:789-795, 1981.

Kokotovic, P. V., Khalil, H. K., and O'Reilly, J. Singular Perturbations in Control: Analysis and Design. Academic Press, London, 1986. 
Kumar, A. and Daoutidis, P. Nonlinear dynamics and control of process systems with recycle. J. Process Contr., 12(4):475-484, 2002.

Kumar, A., Christofides, P. D., and Daoutidis, P. Singular perturbation modeling of nonlinear processes with nonexplicit time-scale multiplicity. Chem. Eng. Sci., 53(8):1491-1504, 1998.

Ladde, G. S. and Siljak, D. D. Multiparameter singular perturbation of linear systems with multiple time scales. Automatica, 19:385-394, 1983.

Larsson, T., Govatsmark, M. S., Skogestad, S., and Yu, C. C. Control structure selection for reactor, separator, and recycle processes. Ind. Eng. Chem. Res., 42(6):1225-1234, 2003.

Liu, J., de la Peña, D. M., and Christofides, P. D. Distributed model predictive control of nonlinear process systems. AIChE J., 55(5):1171-1184, 2009 .

Luyben, M. L., Tyreus, B. D., and Luyben, W. L. Plantwide control design procedure. AIChE J., 43(12):3161-3174, 1997.

Marino, R. and Kokotovic, P. V. A geometric approach to nonlinear singular perturbed control systems. Automatica, 24:31-41, 1988.

O'Malley Jr., R. E. Singular Perturbation Methods for Ordinary Differential Equations. Springer-Verlag, New York, 1991.

Rawlings, J. B. and Stewart, B. T. Coordinating multiple optimizationbased controllers: New opportunities and challenges. J. Process Contr., 18(9):839-845, 2008.

Scattolini, R. Architectures for distributed and hierarchical model predictive control-a review. J. Process Contr., 19(5):723-731, 2009.

Skogestad, S. Control structure design for complete chemical plants. Comput. Chem. Eng., 28(1-2):219-234, 2004.

Stephanopoulos, G. and Ng, C. Perspectives on the synthesis of plant-wide control structures. J. Proc. Contr., 10(2):97-111, 2000.

Sun, Y. and El-Farra, N. H. Quasi-decentralized model-based networked control of process systems. Comput. Chem. Eng., 32(9):2016-2029, 2008.

Tarjan, R. E. Enumeration of the elementary circuits of a directed graph. SIAM J. Comput., 2(3):211-216, 1973.

Venkat, A. N., Hiskens, I. A., Rawlings, J. B., and Wright, S. J. Distributed mpc strategies with application to power system automatic generation control. IEEE T. Contr. Syst. T., 16(6):1192-1206, 2008. 
Vora, N., Contou-Carrere, M., and Daoutidis, P. Model reduction of multiple time scale processes in non-standard singularly perturbed form. In Gorban, A. N., Kevrekidis, I. G., Theodoropoulos, C., Kazantzis, N. K., and ttinger, H. C., editors, Model Reduction and Coarse-Graining Approaches for Multiscale Phenomena, pages 99-113. Springer Berlin Heidelberg, 2006.

Ydstie, B. E. Passivity based control via the second law. Comput. Chem. Eng., 26(7-8):1037-1048, 2002.

Zhu, G. Y., Henson, M. A., and Ogunnaike, B. A. A hybrid model predictive control strategy for nonlinear plant-wide control. J. Process Contr., 10 (5):449-458, 2000. 


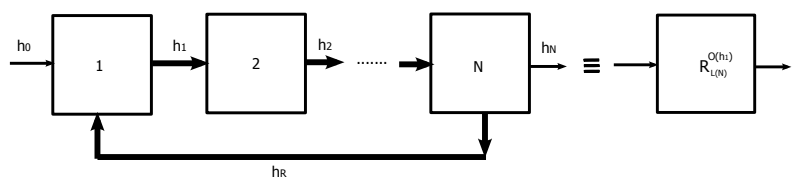

(a) Recycle

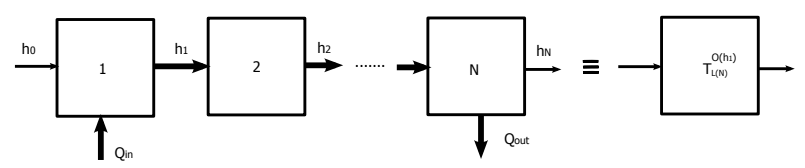

(b) Throughput

Figure 1: Recycles, throughputs and their equivalent representation in energy flow graphs 


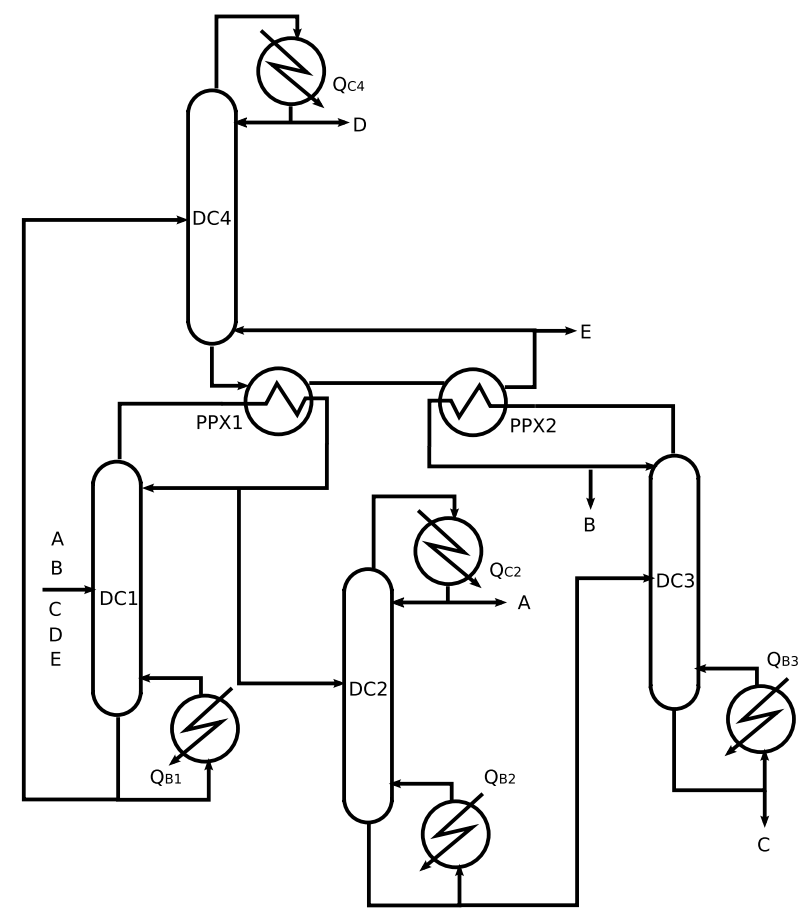

(a) Schematic process flow diagram

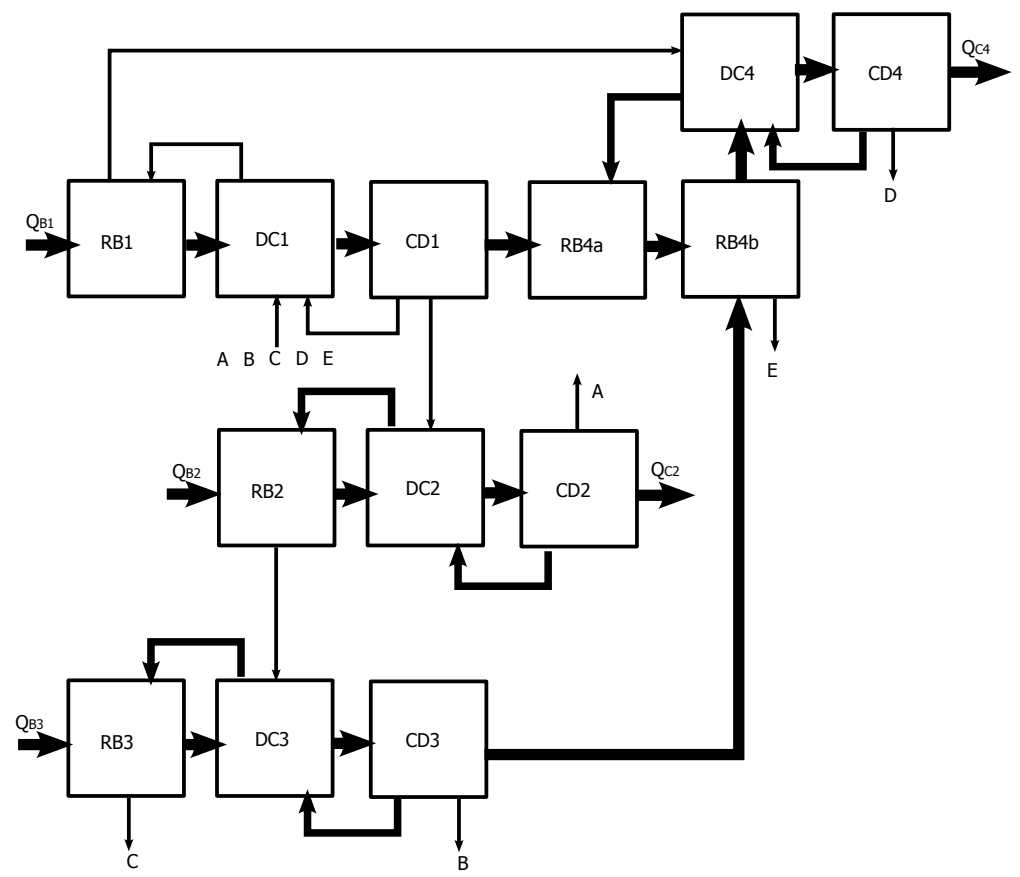

(b) Energy flow diagram (DC: distillation column, RB: reboiler, CD: condenser)

Figure 2: Distillation system for the separation with a mixture of five species 


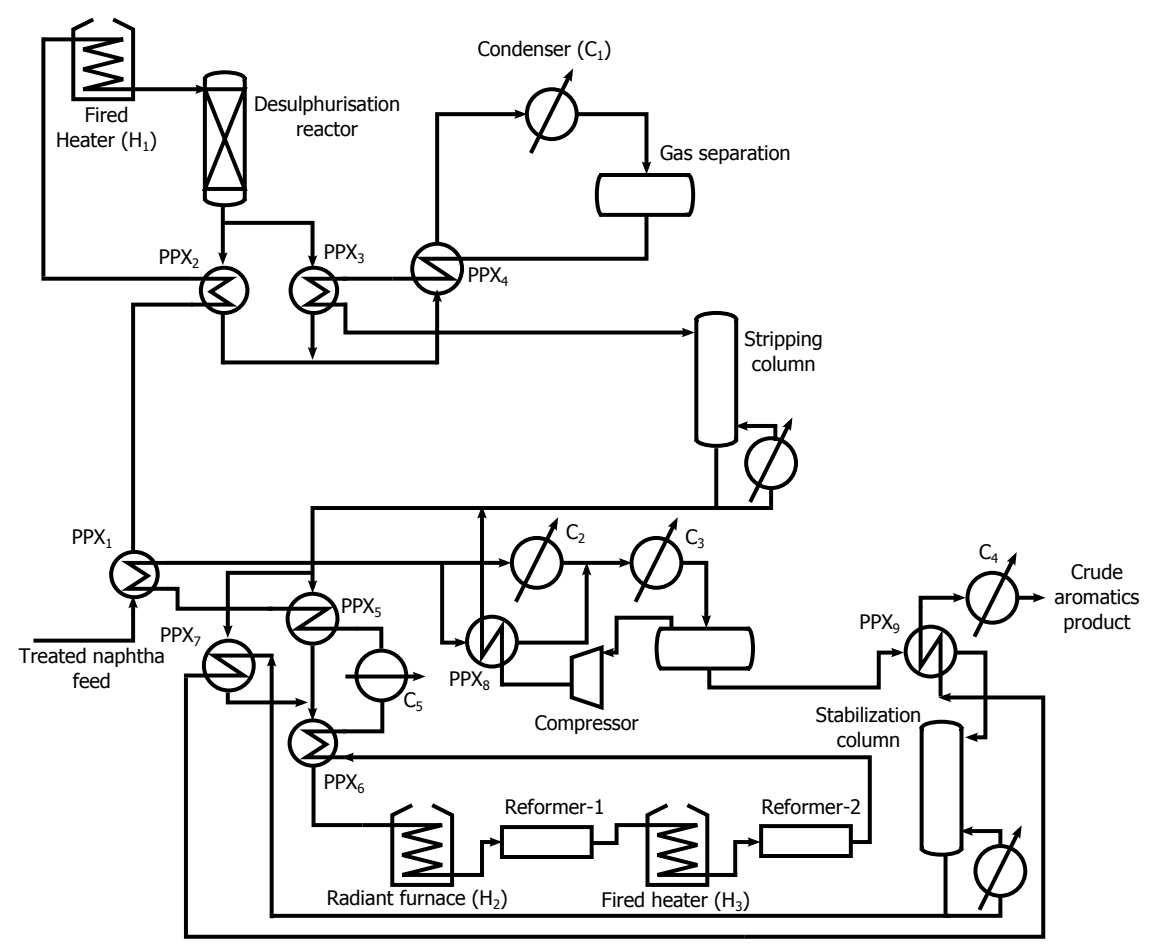

Figure 3: Energy-integrated reactor-heat exchanger network for naphtha reforming


Figure 4: Distinction between a recycle and a bypass 


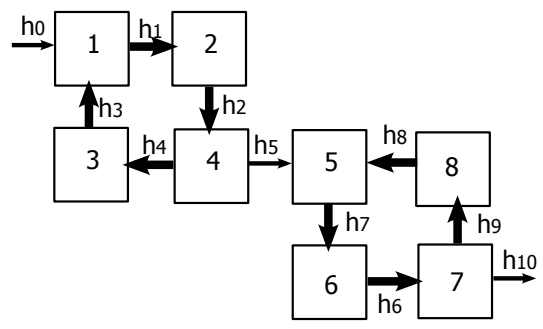

$$
\begin{array}{|l|}
\hline R_{1,2,3,4}^{\mathrm{O}(\mathrm{h} 1)} \\
\text { Subgraph with the } \\
\text { largest magnitude } \\
\text { energy flows }
\end{array}
$$

Figure 5: Energy flow graph for a network with two independent recycles

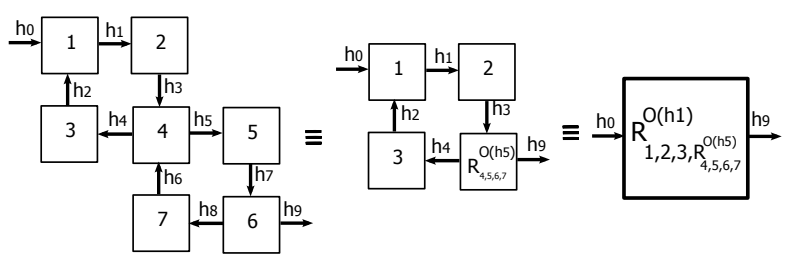

(a) Multiple cycles

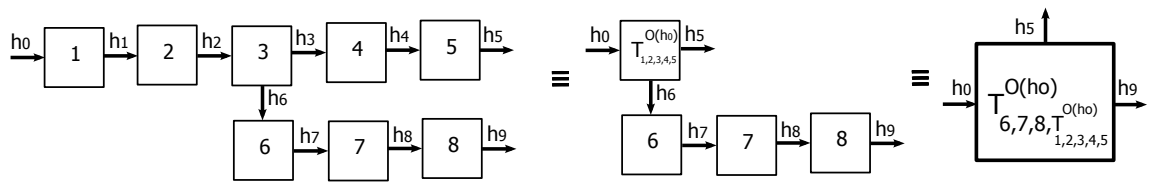

(b) Multiple paths

Figure 6: Process energy flow graphs with multiple paths and cycles 


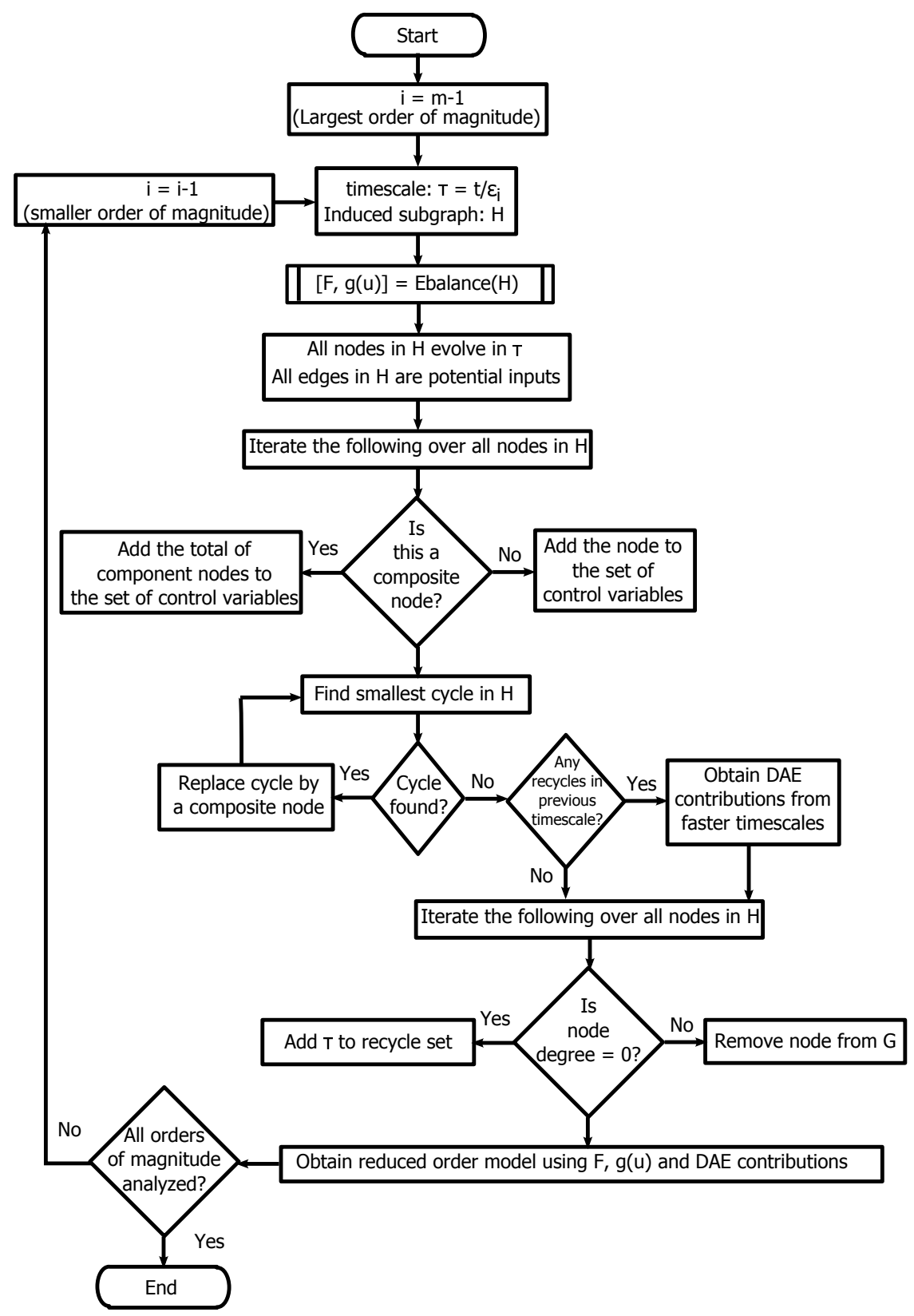

Figure 7: Flowchart for the algorithm ComplexNetworkAnalysis 
Table 1: Node details for the reactor-HE system (DR: Desulphurisation reactor, SC: Stabilization column

\begin{tabular}{|l|l|l|l|}
\hline Index & Node details & Index & Node details \\
\hline \multicolumn{3}{|c|}{ Primary nodes } \\
\hline 1,2 & $P P X_{1}$-cold, hot & 18,19 & $P P X_{7}$-cold, hot \\
3,4 & $P P X_{2}$-cold,hot & 20,21 & $C_{5}, H_{2}$ \\
5 & $H_{1}$ & 22 & Reformer-1 \\
6 & DR & 23 & $H_{3}$ \\
7,8 & $P P X_{3}$-cold, hot & 24 & Reformer-2 \\
9,10 & $P P X_{4}$-cold, hot & 25,26 & $P P X_{8}$-cold, hot \\
11 & $C_{1}$ & 27,28 & $C_{2}, C_{3}$ \\
12 & Gas separation & 29 & Mixer \\
13 & Stripping column & 30 & Compressor \\
14,15 & $P P X_{5}$-cold, hot & 31,32 & $P P X_{9}$-cold, hot \\
16,17 & $P P X_{6}$-cold, hot & 33,34 & $C_{4}$, SC \\
\hline
\end{tabular}

\begin{tabular}{|l|l|l|l|}
\hline \multicolumn{3}{|c|}{ Auxiliary nodes } \\
\hline 35 & $H_{1}$-head & 43 & Stripping column energy input \\
36 & $H_{2}$-head & 44 & Reformer-1 heat of reaction \\
37 & $H_{3}$-head & 45 & Reformer-1 heat of reaction \\
38 & $C_{1}$-tail & 46 & Stabilizing column energy input \\
39 & $C_{2}$-tail & 47 & Compressor power input \\
40 & $C_{3}$-tail & 48 & Desulphurisation heat of reaction \\
41 & $C_{4}$-tail & 49 & Naphtha feed-head \\
42 & $C_{5}$-tail & 50 & Aromatics product-tail \\
\hline
\end{tabular}

Table 2: Nominal values of heat exchanger duties for the naphtha reforming process network

\begin{tabular}{|c|rl||c|rc|}
\hline Exchanger & \multicolumn{2}{|c|}{ Duty } & Exchanger & \multicolumn{2}{|c|}{ Duty } \\
\hline$H_{1}$ & 3.72 & $\mathrm{~kW}$ & $P P X_{1}$ & 4.88 & $\mathrm{~kW}$ \\
$H_{2}$ & 12.55 & $\mathrm{~kW}$ & $P P X_{2}$ & 17.20 & $\mathrm{~kW}$ \\
$H_{3}$ & 37.77 & $\mathrm{~kW}$ & $P P X_{3}$ & 1.88 & $\mathrm{~kW}$ \\
$C_{1}$ & 3.80 & $\mathrm{~kW}$ & $P P X_{4}$ & 8.50 & $\mathrm{~kW}$ \\
$C_{2}$ & 4.77 & $\mathrm{~kW}$ & $P P X_{5}$ & 10.80 & $\mathrm{~kW}$ \\
$C_{3}$ & 6.20 & $\mathrm{~kW}$ & $P P X_{6}$ & 29.30 & $\mathrm{~kW}$ \\
$C_{4}$ & 0.35 & $\mathrm{~kW}$ & $P P X_{7}$ & 4.10 & $\mathrm{~kW}$ \\
$C_{5}$ & 12.50 & $\mathrm{~kW}$ & $P P X_{8}$ & 3.84 & $\mathrm{~kW}$ \\
& & & $P P X_{9}$ & 7.90 & $\mathrm{~kW}$ \\
\hline
\end{tabular}


Table 3: Results of graph-reduction algorithm for the naphtha reforming process network

\begin{tabular}{|c|c|c|c|}
\hline $\begin{array}{c}\text { Time } \\
\text { scale }\end{array}$ & $\mathcal{T}$ & $\mathcal{Y}$ & $\mathcal{U}$ \\
\hline \multirow{5}{*}{$\tau_{5}$} & 3,4, & 16 & $3 \rightarrow 5$ \\
& 5,6, & 17 & $4 \rightarrow 3$ \\
& 16 & 21 & $5 \rightarrow 6$ \\
& 21 & 22 & $6 \rightarrow 4$ \\
& 22 & 23 & $16 \rightarrow 21$ \\
& 24 & any 3 out of & $21 \rightarrow 22$ \\
& & $3,4,5 \& 6$ & $22 \rightarrow 44$ \\
& & & $23 \rightarrow 24$ \\
& & & $24 \rightarrow 17$ \\
& $1,2,3,4$, & $1,2,7,8$, & $17 \rightarrow 3$ \\
& $5,6,7,8$, & $9,10,11,12$, & $2 \rightarrow 1$ \\
$\tau_{1}$ & $9,10,11,12$, & $13,14,15,18$, & $2 \rightarrow 26$ \\
& $13,14,15,18$, & $19,20,25,26$, & $2 \rightarrow 27$ \\
& $19,20,25,26$, & $27,28,29,30$, & $7 \rightarrow 13$ \\
& $27,28,29,30$, & $31,32,33,34$ & $8 \rightarrow 7$ \\
& $31,32,33,34$ & $3+4+5+6$ & $8 \rightarrow 10$ \\
\hline
\end{tabular}

